\title{
Measurement of Mean Raindrop Shape from Polarimetric Radar Observations
}

\author{
EUGENIO GoRguCCI AND GIANFRANCO SCARCHILLI \\ CNR Istituto di Fisica dell Atmosfera, Rome, Italy \\ V. CHANDRASEKAR AND V. N. BRINGI \\ Colorado State University, Fort Collins, Colorado
}

(Manuscript received 6 July 1999, in final form 3 February 2000)

\section{ABSTRACT}

\begin{abstract}
Interpretation of polarimetric radar measurements in rainfall such as differential reflectivity and specific differential phase shifts depends on the mean raindrop shape-size relationship. Currently, semiempirical relations between the oblateness and the diameter of the drop are being used. This paper presents an algorithm to obtain the mean shape of the rain drops from polarimetric radar measurements, namely, the reflectivity factor, the differential reflectivity, and the specific differential phase shift. The accuracy of the estimate mean drop shape depends on the measurement accuracies of polarimetric radar observations. Based on asymptotic error analysis and simulations it is shown that the mean raindrop shape can be estimated to an accuracy of $10 \%$. The raindrop shape estimator algorithm developed in this paper is applied to polarimetric radar data collected by the CSUCHILL radar during the 28 July 1997 Fort Collins, Colorado, flood.
\end{abstract}

\section{Introduction}

The mean shape of raindrops plays a critical role in the interpretation of the polarimetric radar measurements. The mean shape raindrop also plays an important role in the development of algorithms to estimate rainfall rate and liquid water content based on reflectivity factor $\left(Z_{H}\right)$, differential reflectivity $\left(Z_{\mathrm{DR}}\right)$, and specific differential propagation phase $\left(K_{\mathrm{DP}}\right)$. The equilibrium shape of a raindrop, falling at its terminal fall speed, is determined by the balance between the forces due to surface tension, hydrostatic pressure, and aerodynamic pressure from airflow around the drop. The shapes of raindrops have been studied theoretically by Green (1975) and Beard and Chuang (1987), experimentally in wind tunnels by Pruppacher and Beard (1970), and in natural rainfall using aircraft probes by Chandrasekar et al. (1988) and Bringi et al. (1998). The experimental results of Chandrasekar et al. (1988) and Bringi et al. (1998) were fairly consistent with the model results of Beard and Chuang (1987). All of the above studies as well as polarimetric radar measurements at multiple polarizations show that the shape of raindrops can be approximated by an oblate spheroid, described with an

Corresponding author address: Dr. Eugenio Gorgucci, CNR Istituto di Fisica dell'Atmosfera, Area di Ricerca Roma-Tor Vergata, Via del Fosso del Cavaliere, 100-00133 Rome. Italy.

E-mail: gorgucci@radar.ifa.rm.cnr.it axis ratio $(b / a)$ and equivolumetric spherical diameter $D$, where $a$ and $b$ are the major and the minor axes of the drop, respectively. A commonly used approximation relating the axis ratio of a raindrop to the diameter is given by (Pruppacher and Beard 1970):

$$
b / a=1.03-0.062 D .
$$

In addition, nonlinear relations are available to model axis ratios of raindrops (Andsager et al. 1999). The experimental results of Bringi et al. (1998) showed that the axis ratios were higher than the model given by (1) for $D<3 \mathrm{~mm}$. However, for $D>4.5 \mathrm{~mm}$ the mean axis ratios were smaller than those given by (1). The above results were obtained after careful and tedious analysis of aircraft-mounted 2D imaging probe data. It would be very useful to obtain an estimate of the mean shape-size relation from polarimetric radar measurements in order to study any variability in the mean shape of the raindrops in different storms as well as different regions of storms.

The objective of this paper is to derive an algorithm to estimate the mean shape of raindrops from polarimetric radar data. The paper is organized as follows. Section 2 defines the mean shape model for raindrops, whereas section 3 describes the effect of raindrop shape on polarimetric radar measurements. The estimator for mean raindrop shape from radar measurements is developed and its accuracy and sensitivity are evaluated in section 4. The estimator developed in this paper is applied to data collected by the CSU-CHILL radar dur- 
ing the 28 July 1997 Fort Collins, Colorado, flood and the results are presented in section 5. Section 6 summarizes the important results of the paper.

\section{Mean raindrop shape model}

Polarimetric radar measurements, wind tunnel measurements, as well as in situ observations using airborne 2D probes indicate that the shape of raindrops can be approximated by oblate spheroids described by semimajor axis $a$ and semiminor axis $b$. The axis ratio of the raindrop $(r)$ is given by

$$
r=\frac{b}{a} .
$$

The equivolumetric spherical diameter is defined by equating the volume of the spheroid to that of a sphere by

$$
\frac{\pi}{6} D^{3}=\frac{4}{3} \pi a^{2} b .
$$

As noted before, the shape versus size relation can be approximated by a straight line given by

$$
r=1.03-\beta D \text {. }
$$

In Eq. (3) $r=1$ when $D \leq 0.03 / \beta$, where $\beta$ is the magnitude of the slope of the shape-size relationship given by

$$
\beta=-\frac{d r}{d D} .
$$

The approximation given by (1) corresponds to $\beta=$ $0.062 \mathrm{~mm}^{-1}$, which is close to the equilibrium shapesize relation, and therefore we denote it by $\beta_{c}$. We note $\beta>\beta_{e}$ indicates that raindrops are more oblate than equilibrium, whereas $\beta<\beta_{e}$ indicates raindrops are less oblate (or closer to spherical) than equilibrium.

\section{Polarimetric radar measurements: Sensitivity to shape-size relation}

The three commonly used polarimetric radar parameters are reflectivity factor at horizontal polarization $\left(Z_{\mathrm{H}}\right)$, differential reflectivity $\left(Z_{\mathrm{DR}}\right)$, and specific differential propagation phase $\left(K_{\mathrm{DP}}\right)$. Both the cloud model and measurements of raindrop size distribution (RSD) at the surface and aloft show that a gamma distribution model adequately describes many of the natural variations in RSD (Ulbrich 1983):

$$
N(D)=n_{0} f(D) \quad\left(\mathrm{m}^{-3} \mathrm{~mm}^{-1}\right),
$$

where $N(D)$ is the number of raindrops per unit volume per unit size interval, $n_{c}$ is the concentration, and $f(D)$ is the gamma probability density function (pdf), given by

$$
f(D)=\frac{\Lambda^{\mu+1}}{\Gamma(\mu+1)} e^{-\Lambda D} D^{\mu},
$$

where $\Lambda$ and $\mu$ are parameters of the gamma pdf, and
$\Gamma$ indicates gamma function (Abramovitz and Stegun 1970). The parameter $N_{0}$ defined by Ulbrich (1983) is related to $n_{c}$ as

$$
n_{c}=N_{0} \frac{\Gamma(\mu+1)}{\Lambda^{\mu+1}} .
$$

The volume-weighted median drop diameter $D_{0}$ can be defined as

$$
\int_{0}^{D_{0}} D^{3} N(D) d D=\int_{D_{0}}^{\infty} D^{3} N(D) d D .
$$

The diameter $D_{0}$ can be written in terms of the parameters $\Lambda$ and $\mu$ as

$$
D_{0}=\frac{3.67+\mu}{\Lambda} .
$$

The reflectivity factor $Z_{\mathrm{H}, \mathrm{V}}$ at horizontal $(\mathrm{H})$ and vertical (V) polarization can be expressed as

$Z_{\mathrm{H}, \mathrm{V}}=\frac{\lambda^{4}}{\pi^{5}|k|^{2}} \int \sigma_{H, V}(D, \beta) N(D) d D \quad\left(\mathrm{~mm}^{6} \mathrm{~m}^{-3}\right)$,

where $\sigma_{H, V}$ denote the radar cross sections at the two linear polarizations; $\lambda$ is the wavelength; and $k=(\varepsilon$, $-1) /\left(\varepsilon_{r}+2\right)$, where $\varepsilon_{r}$ is the dielectric constant of water. Similarly, the differential reflectivity $\left(Z_{\mathrm{DR}}\right)$ and specific differential phase $\left(K_{\mathrm{DP}}\right)$ can be expressed as

$Z_{\mathrm{DR}}=10 \log _{10} \frac{\int \sigma_{H}(D, \beta) N(D) d D}{\int \sigma_{v}(D, \beta) N(D) d D}$

and

$$
\begin{array}{r}
K_{\mathrm{DP}}=\frac{180 \lambda}{\pi} \Re \int\left[f_{H}(D, \beta)-f_{V}(D, \beta)\right] N(D) d D \\
\left(\mathrm{deg} \mathrm{km}^{-1}\right),
\end{array}
$$

where $f_{H}, f_{V}$ are the forward scatter amplitudes at $H$ and $V$ polarization states. It can be seen from $(10 \mathrm{a}-\mathrm{c})$ that for a given RSD, $Z_{\mathrm{H}}, Z_{\mathrm{DR}}$, and $K_{\mathrm{DP}}$ can change with shape-size relationship for raindrops.

According to (1), raindrops become more oblate when the size is large. Therefore, the effect of varying shapesize relationship should be more evident in the presence of larger drops. The volume-weighted median drop diameter $D_{0}$ is a good indicator of the mean size of drops in the distribution. The effect of varying shape-size relations of raindrops is illustrated by the following analysis. For a given RSD and at S-band frequency, we compute the radar measurements $Z_{\mathrm{H}}, Z_{\mathrm{DR}}$, and $K_{\mathrm{DP}}$ for various $\beta$ in the range of $0.02-0.1$ in steps of 0.01 . The various shape-size relationships studied here are shown in Fig. 1, where the dash-dotted line represents the equi- 


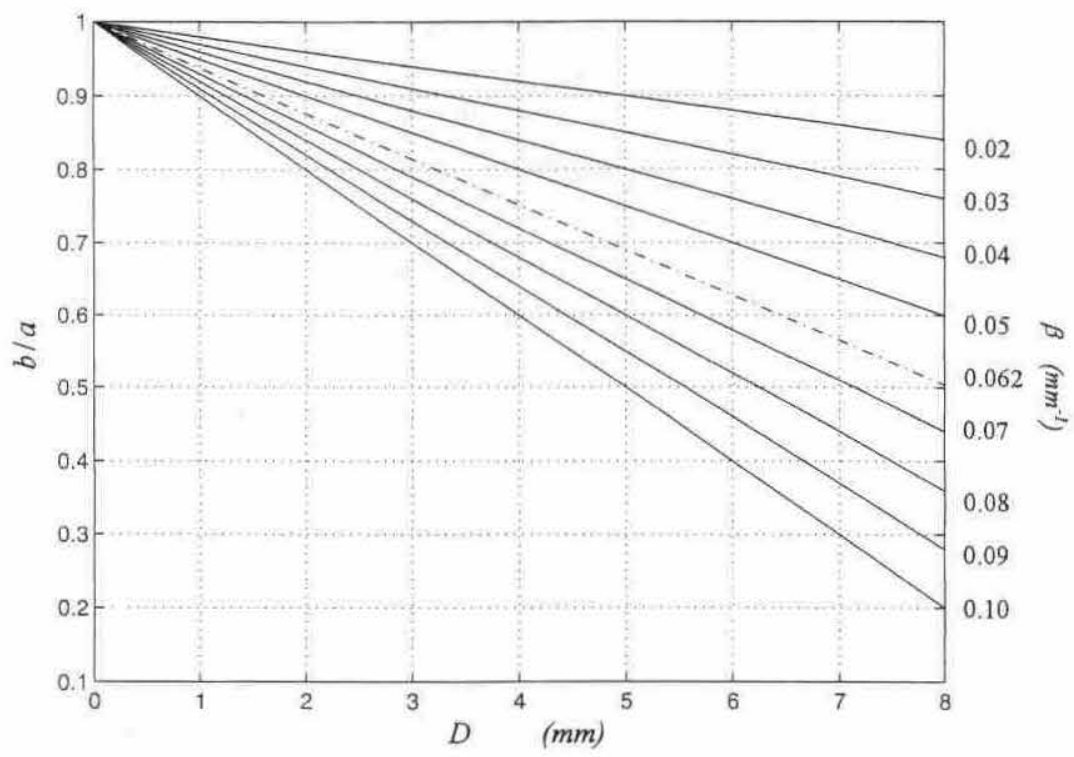

FlG. 1. The raindrop axis ratio $(b / a)$ as a function of the equivolumetric diameter $D$ for different values of the slope $\beta$. The dash-dotted line represents the Pruppacher and Beard relation.

librium relation (1). In Fig. 2 the behavior of $Z_{\mathrm{DR}}$ (in linear scale) is shown as a function of $D_{0}$ for different values of $\beta$. It can be noted that $Z_{\mathrm{DR}}$ increases as $D_{0}$ increases for any value of $\beta$ (Seliga and Bringi 1976); moreover, for a given $D_{0}, Z_{\mathrm{DR}}$ increases with $\beta$. Similar behavior can also be obtained for $K_{\mathrm{DP}}$. As shown in Fig. 2 , the sensitivity of $Z_{D R}$ to $\beta$ is most dependent on $D_{0}$. Figure 3a shows the normalized variation of $Z_{\mathrm{DR}}$ (in linear scale) with respect to $Z_{D R}$ obtained from the equi-

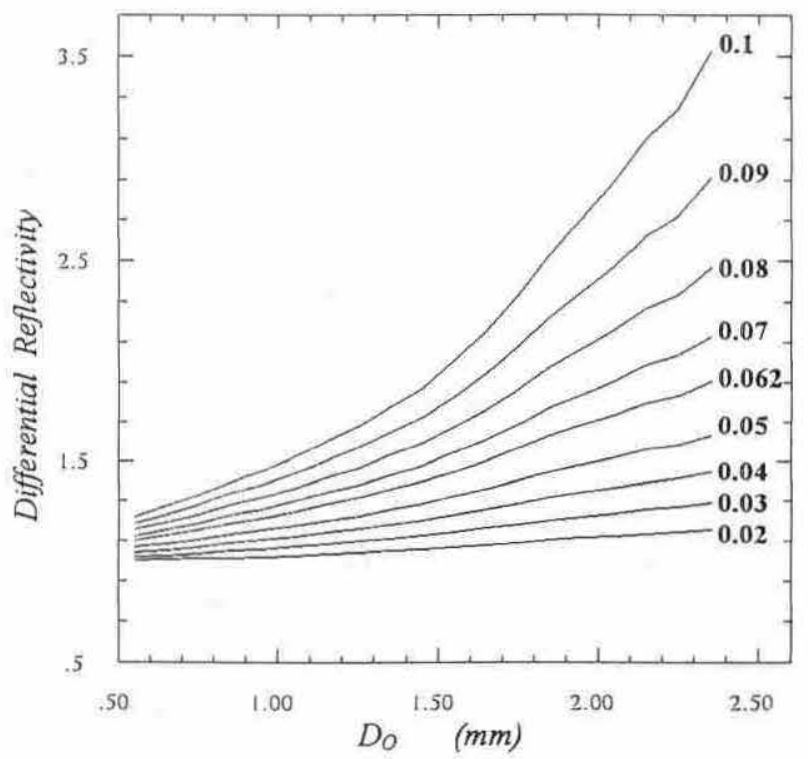

FIG. 2. Averaged value of differential reflectivity (in linear scale), as a function of median drop diameter $\left(D_{0}\right)$ for different values of $\beta$, for various RSD. librium relation (1) as a function of $\beta$ for different values of $D_{0}$. For nearly spherical particles $(\beta \cong 0)$, the $Z_{\mathrm{DR}}$ value should be $0 \mathrm{~dB}$ or unity in linear scale. The normalized bias for $\beta \cong 0$ in comparison to $\beta$ e is determined by the value of $Z_{\mathrm{DR}}$ at $\beta=0.062$ so that it increases as $D_{0}$ increases (see Fig. 2). The range of $Z_{\mathrm{DR}}$ difference between nearly spherical drops $(\beta=0.02)$ and equilibrium-shape drops varies between 0.84 and $1.89 \mathrm{~dB}$ depending on $D_{0}$. Similar arguments can also be made when $\beta>0$ so that normalized bias of $Z_{\mathrm{DR}}$ increases with $D_{0}$ as we move farther from $\beta \cong 0$. Figure $3 \mathrm{~b}$ shows similar analysis for $K_{\mathrm{DP}}$. For nearly spherical particles $(\beta \cong 0)$ and $D_{0} \leq 1 \mathrm{~mm} K_{\mathrm{DP}}$ is approximately zero and then the ratio between $K_{\mathrm{DP}}$ with respect to $K_{\mathrm{DP}}$ at equilibrium axis ratio is nearly zero and the normalized bias has the maximum negative value equal to -1 . By increasing $D_{0}, K_{\mathrm{DP}}$ increases and then the normalized bias decreases. Similar results can be obtained for $\beta>$ 0 so that the normalized bias decreases by increasing $D_{0}$. The reflectivity factor is fairly insensitive to raindrop shape-size relationships for $\beta<\beta_{e}$ as shown in Fig. $3 b$, whereas for $\beta>\beta_{e}$ the change in $Z_{H}$ with $\beta$ is within $10 \%$.

\section{Algorithm to estimate raindrop shape-size relation}

The result of section 3 indicates that the observations of $Z_{\mathrm{DR}}$ and $K_{\mathrm{DP}}$ are sufficiently sensitive to $\beta$, so that it can be turned around into measurement. The estimator of $\beta$ is developed using the following procedure. First, large number of Gamma RSD is simulated over a wide range of the parameters $N_{0}, D_{0}$, and $\mu$, as suggested by 

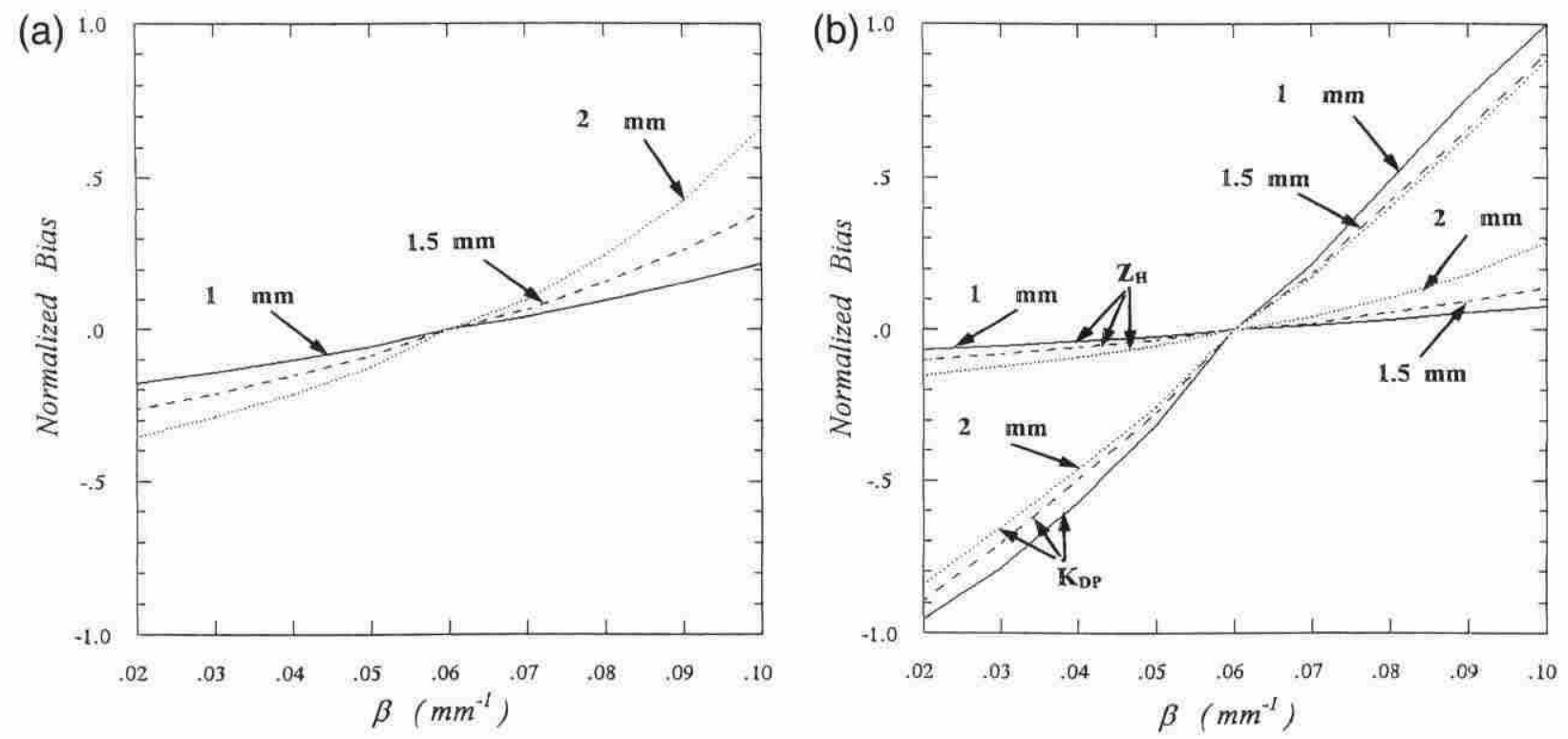

FIG. 3. Normalized bias (a) on the differential reflectivity $\left(Z_{\mathrm{DR}}\right)$, in linear scale, with respect to $Z_{\mathrm{DR}}$, and (b) on the reflectivity factor $\left(Z_{\mathrm{H}}\right)$ and specific differential phase $\left(K_{\mathrm{DP}}\right)$ with respect to $Z_{\mathrm{H}}$ and $K_{\mathrm{DP}}$, obtained from Pruppacher and Beard relation as a function of the slope $\beta$, for the values of the median drop diameter $D_{0}$ corresponding to $1 \mathrm{~mm}$ (solid line), $1.5 \mathrm{~mm}$ (dashed line), and $2 \mathrm{~mm}$ (dotted line).

Ulbrich (1983), chosen randomly in the following intervals:

$$
\begin{aligned}
-1 & <\mu & <4 & \\
10^{3.2+0.216 \mu} & <N_{0}<10^{4.5+0.55 \mu} & & \left(\mathrm{m}^{-3} \mathrm{~mm}^{-1-\mu}\right) \\
0.5 & <D_{0}<2.5 & & (\mathrm{~mm}) .
\end{aligned}
$$

In addition, for each RSD, $Z_{\mathrm{H}}, Z_{\mathrm{DR}}$, and $K_{\mathrm{DP}}$ are computed for various values of $\beta$ ranging between 0.02 and

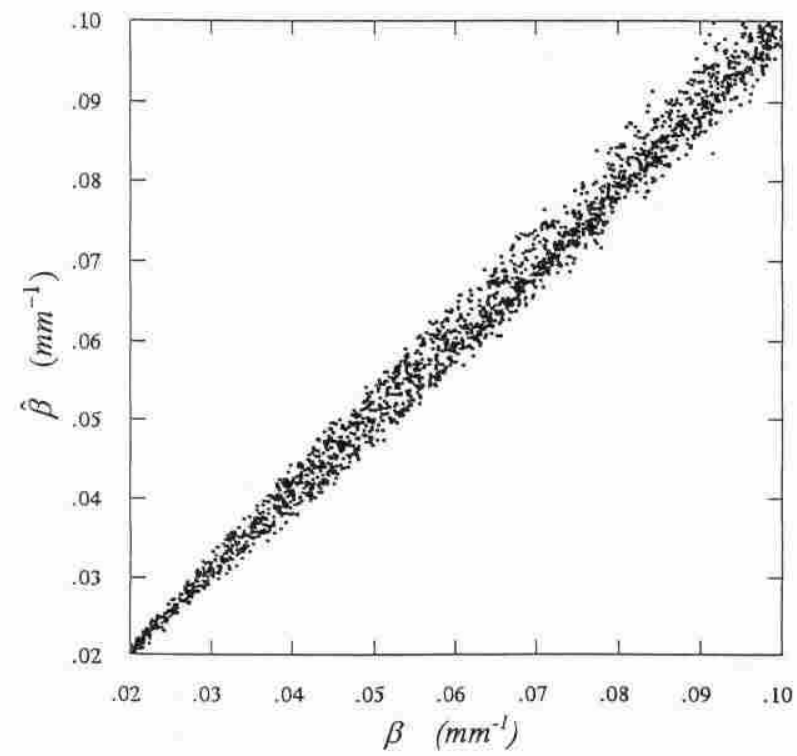

Fig. 4. Scatter diagram between the slope $\beta$ and the estimate $\beta$ computed by (12) in absence of measurement errors on radar observables.
0.1. The above computations are done at S-band frequency. Subsequently, nonlinear regression analysis is performed to evaluate various functional forms to estimate $\beta$. The above analysis yields the estimator for $\beta$ at the S band given (Gorgucci et al. 1999a)

$$
\beta=2.37 Z_{H}^{-0.377} K_{\mathrm{DP}}^{0.396} \times 10^{0.093 Z_{\mathrm{DR}}} .
$$

A scattergram between $\beta$ and the true value of $\beta$ is shown in Fig. 4; it can be noted that (12) estimates $\beta$ fairly well. The data used in Fig. 4 have a correlation of 0.996 with a normalized standard error (the rootmean-square error normalized with the mean) of $3.6 \%$.

\section{a. Shape-size relation estimate in the presence of measurement errors}

The estimate given by (12) uses $Z_{\mathrm{H}}, Z_{\mathrm{DR}}$, and $K_{\mathrm{DP}}$. These three measurements have completely different error structure. The $Z_{\mathrm{H}}$ is based on absolute power measurement and has a typical accuracy of $1 \mathrm{~dB}$. The $Z_{\mathrm{DR}}$ is a relative power measurement and is the differential power estimate between $Z_{\mathrm{H}}$ and $Z_{\mathrm{V}}$. It can be estimated to an accuracy of $0.2 \mathrm{~dB}$. The $K_{\mathrm{DP}}$ is the slope of the range profile of the differential propagation phase $\Phi_{\mathrm{DP}}$, which can be estimated to an accuracy of a few degrees. The subsequent estimate of $K_{\mathrm{DP}}$ depends on the procedure used such as a simple finite-difference scheme or a least squares fit. Using a least squares estimate of the $\Phi_{\mathrm{DP}}$ profile, the standard deviation of $K_{\mathrm{DP}}$ can be expressed as (Gorgucci et al. 1999b)

$$
\sigma\left(K_{\mathrm{DP}}\right)=\sqrt{3} \frac{\sigma\left(\Phi_{\mathrm{DP}}\right)}{N \Delta r} \sqrt{\frac{N}{(N-1)(N+1)}},
$$




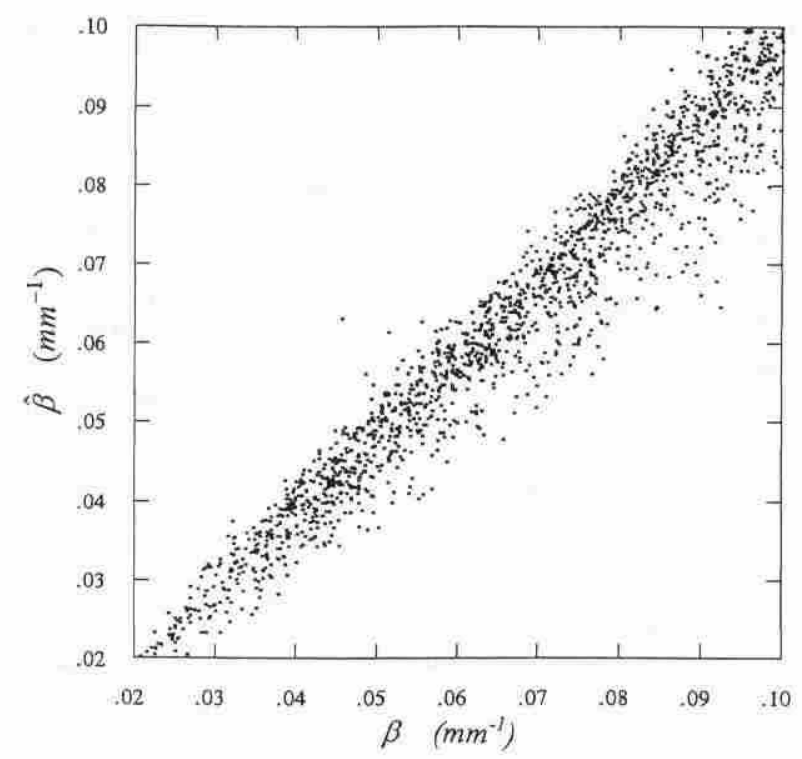

FIG. 5. Scatter diagram between the slope $\beta$ and the estimate $\beta$ computed by (12) in presence of measurement errors on radar observables.

where $\Delta r$ is the range resolution of the $\Phi_{\mathrm{DP}}$ estimate and $N$ is the number of range samples within the path. For large $N$ we can see that $\sigma\left(K_{\mathrm{DP}}\right)$ decreases as $N^{-3 / 2}$. For a typical $150-\mathrm{m}$ range spacing, and with $2.5^{\circ}$ accuracy of $\Phi_{\mathrm{DP}}$, the $K_{\mathrm{DP}}$ can be estimated, over a path of $3 \mathrm{~km}$, with a standard error of $0.32^{\circ} \mathrm{km}^{-1}$. Thus, the three measurements $Z_{\mathrm{H}}, Z_{\mathrm{DR}}$, and $K_{\mathrm{DP}}$ have completely different error structure. In addition, the measurement errors of $Z_{\mathrm{H}}, Z_{\mathrm{DR}}$, and $K_{\mathrm{DP}}$ are nearly independent. In the following we use simulations to quantify the error structure of the estimate of $\beta$. The simulation is done as follows. Various rainfall values are simulated varying the parameters of the gamma RSD over a wide range of values, as suggested by Ulbrich (1983). For each RSD the corresponding $Z_{\mathrm{H}}, Z_{\mathrm{DR}}$, and $K_{\mathrm{DP}}$ are evaluated using $(10 \mathrm{a}-\mathrm{c})$. The random measurement errors are simulated using the procedure described in Chandrasekar et al. (1986). The principal parameters of our simulation are as follows: 1) wavelength $\lambda=11 \mathrm{~cm} ; 2$ ) sampling time PRT $=1 \mathrm{~ms}$; 3) number of samples pairs $M=64$; 4) Doppler velocity spectrum width $\sigma_{v}=2 \mathrm{~m} \mathrm{~s}^{-1} ; 5$ ) cross correlation between $\mathrm{H}$ and $\mathrm{V}$ signals $\rho_{\mathrm{HV}}=0.99 ; 6$ ) range sample spacing over the path where $K_{\mathrm{DP}}$ is estimated is $150 \mathrm{~m}$; and 7) $K_{\mathrm{DP}}$ is estimated over a path of 50 range samples, as a least squares fit on $\Phi_{\mathrm{DP}}$ measurements. Figure 5 shows the scatter diagram of $\beta$ given by (12) versus $\beta$ in the presence of measurement errors, using $K_{\mathrm{DP}}$ values greater than $0.4^{\circ} \mathrm{km}^{-1}$. The scatter diagram of the data in Fig. 5 gives a correlation coefficient of 0.97 and a normalized standard error of $9 \%$. Finally, Fig. 6 shows the normalized standard error of $\beta$ as a function of $\beta$, where normalized standard error is defined as the root-mean-square error normalized with respect to the mean. The results of Fig. 6 show that the

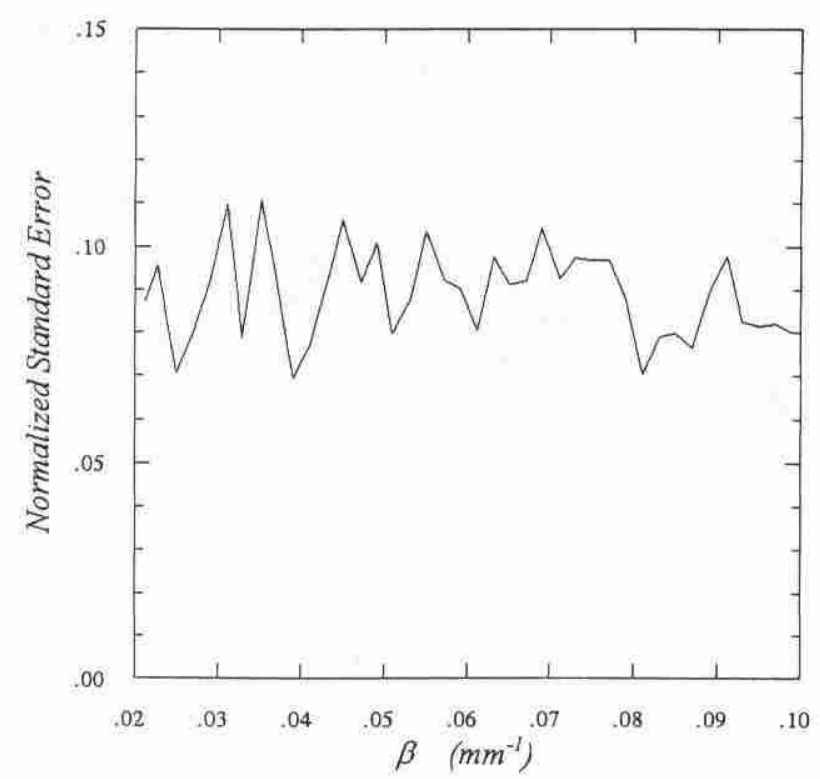

FIG. 6. Normalized standard error of the estimate $\beta$ computed by (12) as a function of the slope $\beta$.

slope of the shape-size relation $\beta$ can be estimated to an accuracy of about $9 \%$ in the presence of measurement errors in $Z_{\mathrm{H}}, Z_{\mathrm{DR}}$, and $K_{\mathrm{Dp}}$. The appendix shows variance computations of $\beta$ only due to measurement errors.

\section{b. Sensitivity of mean shape estimation to bias in $Z_{H}$ and $Z_{D R}$}

Bias errors in $Z_{\mathrm{H}}$ and $Z_{\mathrm{DR}}$ can affect the estimate of $\beta$. Bias errors in the measurements of $Z_{\mathrm{H}}$ and $Z_{\mathrm{DR}}$ will remain even if extensive averaging is performed. The term $Z_{\mathrm{DR}}$ is a differential power measurement and its bias can be estimated and removed easily (Gorgucci et al. 1999b). However, $Z_{\mathrm{H}}$ is based on absolute power measurement and it is difficult to get the absolute calibration. Typically this can be known to an accuracy of $1 \mathrm{~dB}$. Thus $K_{\mathrm{DP}}$ is based on phase measurement and is immune to calibration biases in fairly uniform rain medium. The bias in $\beta$ due to bias errors in $Z_{\mathrm{H}}$ and $Z_{\mathrm{DR}}$ can be defined as

$$
\operatorname{bias}(\hat{\beta})=\frac{E\left[\hat{\beta}\left(\text { with bias in } Z_{\mathrm{H}}, Z_{\mathrm{DR}}\right)\right]}{E\left[\hat{\beta}\left(\text { with no bias in } Z_{\mathrm{H}}, Z_{\mathrm{DR}}\right)\right]} .
$$

Figure 7 shows contours of bias in the estimate of $\beta$ as a function of bias in $Z_{\mathrm{H}}$ and $Z_{\mathrm{DR}}$. The contours line marked 1 indicates no bias, and lines marked different from 1 indicate overestimation $(>1)$ and underestimation $(<1)$. Typically in a well-maintained system, bias error in $Z_{\mathrm{DR}}$ is less than $0.2 \mathrm{~dB}$ and bias in $Z_{\mathrm{H}}$ is less than $1 \mathrm{~dB}$. Therefore, from Fig. 7 it can be seen that $\beta$ can be estimated within $10 \%$ accuracy under those biases of $Z_{\mathrm{H}}$ and $Z_{\mathrm{DR}}$. 


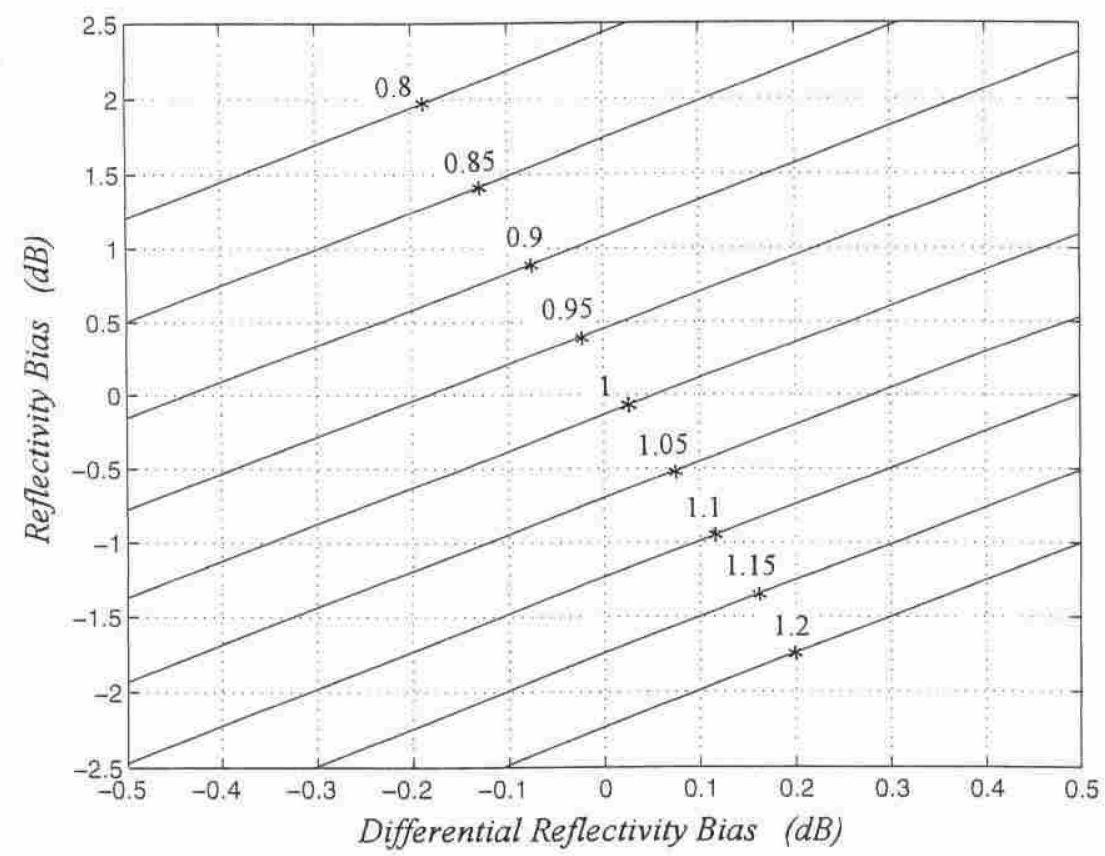

FIG. 7. Contours of bias in the estimate of the slope $\beta$ as a function of biases in the reflectivity $\left(Z_{\mathrm{H}}\right)$ and differential reflectivity $\left(\mathrm{Z}_{\mathrm{DR}}\right)$. The contour line marked 1 indicate no bias, whereas lines marked $>1$ indicate overestimation and $<1$ underestimation, respectively.

\section{Data analysis}

On the evening of 28 July 1997 , the city of Fort Collins was hit by a flash flood that caused fatalities and extensively property damage. Mesoscale analysis of this flood is described in Petersen et al. (1999). CSUCHILL radar recorded continuous data over the event, collecting multiparametric measurements over $5 \mathrm{~h}$. The radar recorded measurements of $Z_{\mathrm{H}}, Z_{\mathrm{DR}}$, and $K_{\mathrm{DP}}$. The characteristics of the CSU-CHILL radar that are rele-

\section{TABLE 1. System characteristics of the CSU-CHILL radar.}

Antenna type

Antenna size

3-dB beamwidth

Gain

Sidelobe level

Polarization radiated

Feed

Wavelength

Peak power

Pulse width

PRT

Type

Max unambiguous range

Max unambiguous velocity

Noise figure

Transfer function

Dynamic range

Min detectable signal vant for this paper are listed in Table 1. The application of algorithm (12) is fairly straightforward, but numerous details are important. A linear least squares fit was done on the $\Phi_{\mathrm{DP}}$ observations to obtain one $\mathrm{K}_{\mathrm{DP}}$ estimate for a 3-km path, whereas $Z_{\mathrm{H}}$ and $Z_{\mathrm{DR}}$ are computed as the mean value of $Z_{\mathrm{H}}$ and $Z_{\mathrm{DR}}$ measurements on the same path. These values of $Z_{\mathrm{H}}, Z_{\mathrm{DR}}$, and $K_{\mathrm{DP}}$ were used in (12) to estimate $\beta$. Only data from regions with $K_{\mathrm{DP}}>$ $0.4^{\circ} \mathrm{km}^{-1}$ were used to ensure good accuracy in the estimate of $\beta$. A histogram of the various observed values of $\beta$, for reflectivity in the range of 40 to $45 \mathrm{dBZ}$, is shown in Fig. 8a, where the mean and standard deviation of data are 0.061 and 0.01 respectively. The standard deviation of data in Fig. 8a is fairly close to measurement standard deviation, as shown in the appendix. Therefore, most of the spread in $\beta$ is due to measurement error. In addition, it can be seen that the mean slope of shape-size relation shown in Fig. 8a is close to the theoretical predictions as well as to experimental observation reported in the literature so far (Beard and Chuang 1987; Chandrasekar et al. 1988; Bringi et al. 1998). Figure $8 \mathrm{~b}$ shows similar results for data corresponding to $45 \mathrm{dBZ}<Z_{\mathrm{H}}<50 \mathrm{dBZ}$. The mean $\beta$ and standard deviation of the data shown in Fig. $8 \mathrm{~b}$ are 0.057 and 0.008 , respectively. Once again it can be seen that most of the standard deviation is due to measurement error, and the mean $\beta$ of 0.057 indicates that the drops are less oblate than $\beta_{e}$ perhaps due to drop oscillations (Beard et al. 1983). Similar stratification was continued for reflectivity ranging between 50 and $53 \mathrm{dBZ}$ and for $Z_{\mathrm{H}}>53 \mathrm{dBZ}$. Figure $8 \mathrm{c}$ shows 

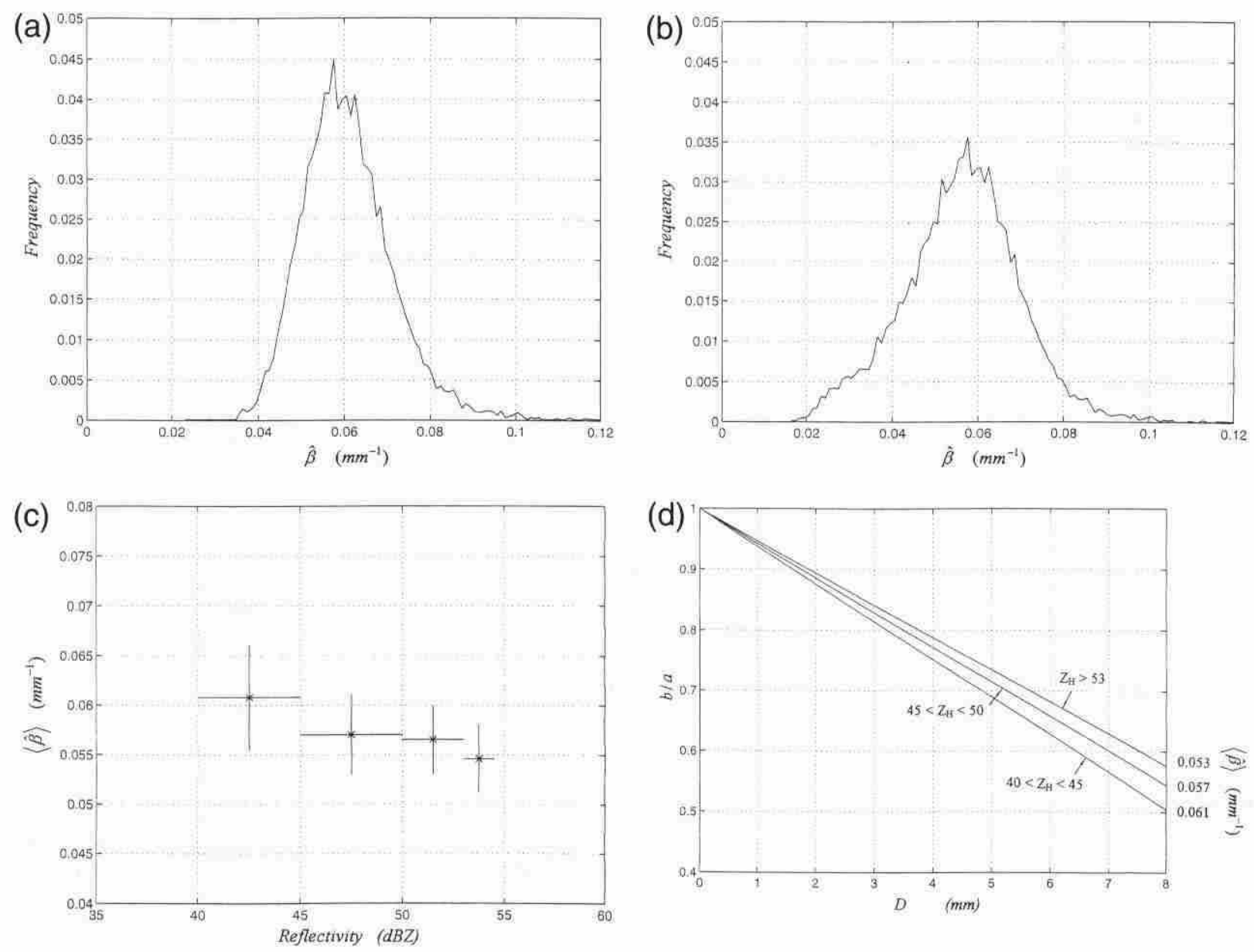

FIG. 8. (a) Histogram of observed values of the estimate $\beta$, computed by (12), for reflectivity factor ranging between 40 and $45 \mathrm{dBZ}$. The data referring to a flash flood that occurred over Fort Collins were collected by the Doppler and polarimetric CSU-CHILL radar. (b) Histogram of observed values of the estimate $\beta$, computed by (12), for reflectivity factor ranging between 45 and $50 \mathrm{dBZ}$. The data referring to a flash flood that occurred over Fort Collins were collected by the Doppler and polarimetric CSU-CHILL radar. (c) The mean value of the estimate $\beta$, signed by star, and the corresponding standard deviation for reflectivity intervals $40-45,45-50$, and $50-53 \mathrm{dBZ}$ and for reflectivity greater than $53 \mathrm{dBZ}$. The data referring to a flash flood that occurred over Fort Collins were collected by the Doppler and polarimetric CSU-CHILL radar. (d) Observed shape-size relation for the values of mean $\beta$ computed for the reflectivity intervals corresponding to $40<Z_{\mathrm{H}}<45$ $\mathrm{dBZ}, 45<Z_{\mathrm{H}}<50$, and for $Z_{\mathrm{H}}>53 \mathrm{dBZ}$. The data referring to a flash flood that occurred over Fort Collins were collected by the Doppler and polarimetric CSU-CHILL radar.

the estimate of mean $\beta$ and its standard deviation as a function of reflectivity. The standard deviation was computed for each case and was found to range between $18 \%$ and $13 \%$ around the mean. Figure 8d shows the observed mean shape-size relations, stratified with reflectivity. It can be seen from Figs. $8 \mathrm{c}$ and $8 \mathrm{~d}$ that the axis ratios become progressively slightly less oblate in comparison to equilibrium axis ratios probably due to raindrop oscillations.

\section{Summary and conclusions}

The mean shape-size relation of raindrops plays an important role in the interpretation of polarimetric radar measurements. The polarimetric radar algorithms available in the literature have been developed for equilibrium axis ratios. A simple model was developed to de- scribe the shape-size relation of raindrops in terms of the slope $(\beta)$ of the linear approximation to the shapesize function. Subsequently, theoretical analysis was utilized to quantify the variability in $Z_{\mathrm{H}}, Z_{\mathrm{DR}}$, and $K_{\mathrm{DP}}$ due to changes in $\beta$. The sensitivity of $Z_{\mathrm{H}}, Z_{\mathrm{DR}}$, and $K_{\mathrm{DP}}$ to deviation from equilibrium shape-size relation $\beta_{e}$ was studied. It was found that both $Z_{\mathrm{DR}}$ and $K_{\mathrm{DP}}$ were fairly sensitive to changes in $\beta$, whereas $Z_{\mathrm{H}}$ was insensitive as expected. There was enough sensitivity to $\beta$ in $Z_{\mathrm{DR}}$ and $K_{\mathrm{DP}}$ that it could be turned around to a measurement. An algorithm to estimate the slope of the shape-size relation was derived. The algorithm can be used to estimate $\beta$ from measurements of $Z_{\mathrm{H}}, Z_{\mathrm{DR}}$, and $K_{\mathrm{DP}}$. Error analysis of the algorithm demonstrated that the algorithm estimates $\beta$ on the average to an accuracy of $9 \%$, when $K_{\mathrm{DP}}$ is estimated over a path of 50 range bins with a range spacing of $150 \mathrm{~m}$. Polarimetric radar data col- 
lected by the CSU-CHILL radar was used to evaluate the algorithm developed in this paper. The estimation of $\beta$ from radar data yielded values very close to the equilibrium shape-size relation of raindrops. When the data were stratified with reflectivity, the results indicated that the drops became less oblate as reflectivity increases, an indication of possible raindrop oscillation.

Acknowledgments. This project was supported by the National Science Foundation (ATM-9413453), by the National Group for Defense from Hydrological Hazards (CNR, Italy), by Progetto Strategico Mesoscale Alpine Program (CNR, Italy), by the Italian Space Agency (ASI), and by the NASA TRMM program. The CSUCHILL is supported by the National Science Foundation (ATM-9500108). The gauge data were collected and archived by the Colorado Climate Center, and the radar data were collected by Bob Bowie of the CSU-CHILL facility. The authors are grateful to A. Mura and P. Iacovelli for assistance rendered during the preparation of the manuscript.

\section{APPENDIX}

\section{Variance in the Estimate of Mean Shape-Size Relation $(\boldsymbol{\beta})$}

The estimate for $\beta$ is given by

$$
\beta=c Z_{\mathrm{H}}^{a_{1}} K_{\mathrm{DP}}^{a_{2}} \times 10^{-a_{3} Z_{\mathrm{DR}}},
$$

where $a_{1}, a_{2}, a_{3}$, and $c$ are the coefficients given by (12). The variance of $\beta$ normalized to the mean value can be expressed from perturbation analysis as

$$
\begin{aligned}
\frac{\operatorname{var}(\hat{\beta})}{\hat{\beta}^{2}}= & a_{1}^{2} \frac{\operatorname{var}\left(Z_{\mathrm{H}}\right)}{Z_{\mathrm{H}}^{2}}+a_{2}^{2} \frac{\operatorname{var}\left(K_{\mathrm{DP}}\right)}{K_{\mathrm{DP}}^{2}} \\
& +\left(a_{3} \ln 10\right)^{2} \operatorname{var}\left(Z_{\mathrm{DR}}\right) .
\end{aligned}
$$

Note that $Z_{\mathrm{H}}$ can be measured to an accuracy of better than $1 \mathrm{~dB}, Z_{\mathrm{DR}}$ can be measured to an accuracy of 0.2 $\mathrm{dB}$, and standard deviation in the estimate of $K_{\mathrm{DP}}$ is given by (12). Assuming 20 range bins with range spacing of $0.15 \mathrm{~km}$ and for a mean value of $K_{\mathrm{DP}}$ of $0.86^{\circ}$ $\mathrm{km}^{-1}$, the normalized standard error (standard deviation normalized with respect to the mean) of $\beta$ is $15 \%$.

\section{REFERENCES}

Abramovitz, M., and A. Stegun, 1970: Handbook of Mathematical Functions. Dover, 1043 pp.

Andsager, K., K. V. Beard, and N. F. Laird, 1999: Laboratory measurements of axis ratios for large raindrops. J. Atmos. Sci., 56, 2673-2683.

Beard, K. V., and C. Chuang, 1987: A new model for the equilibrium shape of raindrops. J. Atmos. Sci., 44, 1509-1524.

- D. B. Johnson, and A. R. Jameson, 1983: Collisional forcing of raindrop oscillations. J. Atmos, Sci., 40, 455-462.

Bringi, V. N., V. Chandrasekar, and R. Xiao, 1998: Raindrop axis ratio and size distributions in Florida rainshafts: An assessment of multiparameter radar algorithms. IEEE Trans. Geosci. Remote Sens., 36, 703-715.

Chandrasekar, V., V. N. Bringi, and P. J. Brockwell, 1986: Statistical properties of dual polarized radar signals. Preprints, 23rd Conf. on Radar Meteorology, Snowmass, CO, Amer. Meteor. Soc., 154-157.

- W. A. Cooper, and V. N. Bringi, 1988: Axis ratios and oscillation of raindrops. J. Atmos. Sci., 45, 1325-1333.

Gorgucci, E., G. Scarchilli, and V. Chandrasekar, 1999a: Estimation of mean raindrop shape from polarimetric radar measurements. Preprints, 29th Int. Conf. on Radar Meteorology, Montreal, PQ, Canada, Amer. Meteor. Soc., 168-171.

$\ldots,-$ and $1999 \mathrm{~b}:$ A procedure to calibrate multiparameter weather radar using properties of the rain medium. IEEE Trans. Geosci. Remote Sens., 37, 269-276.

Green, A. W., 1975: An approximation for the shapes of large raindrops. J. Appl. Meteor., 14, 1578-1583.

Petersen, A. P., and Coauthors, 1999: Mesoscale and radar observations of the Fort Collins flash flood of 28 July 1997. Bull. Amer. Meteor. Soc., 80, 191-216.

Pruppacher, H. R., and K. V. Beard, 1970: A wind tunnel investigation of the internal circulation and shape of water drops falling at terminal velocity in air. Quart. J. Roy. Meteor. Soc, 96, 247256.

Seliga, T. A., and V. N. Bringi, 1976: Potential use of the radar reflectivity at orthogonal polarizations for measuring precipitation. J. Appl. Meteor., 15, 69-76.

Ulbrich, C. W., 1983: Natural variations in the analytical form of raindrop size distributions. J. Climate Appl. Meteor., 22, 1764 1775 . 